\title{
Interobserver reliability in the histopathological diagnosis of cartilaginous tumors in patients with multiple osteochondromas
}

Carlos E de Andrea ${ }^{1,2}$, Herman M Kroon ${ }^{3}$, Ron Wolterbeek ${ }^{4}$, Salvatore Romeo ${ }^{5}$, Andrew E Rosenberg ${ }^{6}$, Barry R De Young ${ }^{7}$, Bernadette Liegl ${ }^{8}$, Carrie Y Inwards ${ }^{9}$, Esther Hauben ${ }^{10}$, Edward F McCarthy ${ }^{11}$, Miguel Idoate ${ }^{2}$, Nicholas A Athanasou ${ }^{12}$, Kevin B Jones ${ }^{13}$, Pancras CW Hogendoorn ${ }^{1}$ and Judith VMG Bovée ${ }^{1}$

${ }^{1}$ Department of Pathology, Leiden University Medical Center, Leiden, The Netherlands; ${ }^{2}$ Department of Pathology, University of Navarra, Pamplona, Spain; ${ }^{3}$ Department of Radiology, Leiden University Medical Center, Leiden, The Netherlands; ${ }^{4}$ Department of Statistics, Leiden University Medical Center, Leiden, The Netherlands; ${ }^{5}$ Department of Pathology, Treviso Regional Hospital, Treviso, Italy; ${ }^{6}$ Department of Pathology, University of Miami Hospital, Miami, FL, USA; ${ }^{7}$ Department of Pathology, University of Iowa Carver College of Medicine, Iowa City, IA, USA; ${ }^{8}$ Institute of Pathology, Medical University of Graz, Graz, Austria; ${ }^{9}$ Department of Laboratory Medicine and Pathology, Mayo Clinic, Rochester, MN, USA; ${ }^{10}$ Department of Pathology, University Hospitals Leuven, Leuven, Belgium; ${ }^{11}$ Department of Pathology and Orthopaedic Surgery, The Johns Hopkins Hospital and Johns Hopkins University School of Medicine, Baltimore, MD, USA; ${ }^{12}$ Nuffield Department of Orthopaedics, Rheumatology and Musculoskeletal Sciences, University of Oxford, Nuffield Orthopaedic Centre, Oxford, UK and ${ }^{13}$ Department of Orthopaedic Surgery, Center for Children's Cancer Research, Huntsman Cancer Institute, Primary Children's Medical Center, University of Utah, Salt Lake City, UT, USA

The distinction between benign and malignant cartilaginous tumors located peripherally in the bone may be a challenging task in surgical pathology. The aim of this study was to investigate interobserver reliability in histological diagnosis of cartilaginous tumors in the setting of multiple osteochondromas and to evaluate possible histological parameters that could differentiate among osteochondroma, low- and high-grade secondary peripheral chondrosarcoma. Interobserver reliability was assessed by 12 specialized bone-tumor pathologists in a set of 38 cases. Substantial agreement on diagnosis among all the reviewers was observed (intraclass correlation coefficient $=\mathbf{0 . 7 8}$ ). Our study confirmed that mitotic figures and nuclear pleomorphism are hallmarks of high-grade secondary peripheral chondrosarcoma. However, despite the substantial agreement, we demonstrated that histology alone cannot distinguish osteochondroma from low-grade secondary peripheral chondrosarcoma in the setting of multiple osteochondromas, as nodularity, the presence of binucleated cells, irregular calcification, cystic/mucoid changes and necrosis were not helpful to indicate malignant transformation of an osteochondroma. On the other hand, among the concordant cases, the cartilage cap in osteochondroma was significantly less thicker than in low- and high-grade secondary peripheral chondrosarcoma. Therefore, our study showed that a multidisciplinary approach integrating clinical and radiographical features and the size of the cartilaginous cap in combination with a histological assessment are crucial to the diagnosis of cartilaginous tumors.

Modern Pathology (2012) 25, 1275-1283; doi:10.1038/modpathol.2012.78; published online 4 May 2012

Keywords: bone tumors; peripheral; cartilage tumors; chondrosarcoma; diagnostic criteria; osteochondroma

Correspondence: Dr JVMG Bovée, MD, PhD, Department of Pathology, Leiden University Medical Center, PO Box 9600, L1-Q, 2300 RC Leiden, The Netherlands.

E-mail: J.V.M.G.Bovee@lumc.nl

Received 7 February 2012; revised 1 March 2012; accepted 1 March 2012; published online 4 May 2012
Multiple osteochondromas, alternatively called multiple hereditary exostoses, is an autosomal dominant disorder with a prevalence estimated at 1 in $50000,{ }^{1}$ and is caused by mutations in EXT1 at 
8q24 or EXT2 at 11p11-13. ${ }^{2}$ Multiple osteochondromas develop during skeletal growth and is characterized by shortened long bones and formation of multiple cartilage-capped bony projections from the metaphyses of endochondral bones adjacent to the growth plate. ${ }^{3}$ Osteochondroma can eventually transform into a secondary peripheral chondrosarcoma in $1-3 \%$ of patients with multiple osteochondromas. $^{2}$

Secondary peripheral chondrosarcomas are malignant cartilage-producing tumors and comprise $\sim 15 \%$ of all conventional chondrosarcomas in tertiary referral centers. ${ }^{4}$ Patients with secondary peripheral chondrosarcomas show a diverse clinical course, ranging from slow insidious tumor growth to rapid neoplastic progression, especially when located in pelvis, shoulder and hip. ${ }^{5}$ Secondary peripheral chondrosarcomas are often low-grade malignant tumors, ie, grade-I chondrosarcomas according to the Evans grading system, ${ }^{6}$ and are treated by limb salvage surgery with a wide or even with a marginal resection. ${ }^{7}$

The lack of consistent and reproducible criteria to determine neoplastic transformation in multiple osteochondromas often raises diagnostic dilemmas in differentiating osteochondroma from low-grade secondary peripheral chondrosarcoma. The malignant potential of an osteochondroma is often estimated by the thickness of its cartilage cap and the evidence of tumor growth in a skeletally mature patient. ${ }^{8}$ Cap thickness greater than $2 \mathrm{~cm}$ in patients with fused growth plates should be considered suspicious for progression of an osteochondroma to a chondrosarcoma. ${ }^{9}$ Moreover, cap thickness greater than $2 \mathrm{~cm}$ strongly indicates secondary peripheral chondrosarcoma. ${ }^{10,11}$ Additionally, a wide range of histological parameters is described to determine malignant transformation of an osteochondroma, ranging from the formation of nodules to the presence of mitotic figures and cystic cavities. ${ }^{12}$ For a general pathologist, the application of these criteria is often difficult, which gives room for a subjective interpretation. Therefore, identifying stringent and reproducible histological criteria may help the interpretation of peripheral cartilaginous tumors by general pathologists.

As no 'gold standard' exists to directly assess tumor grade, prediction of peripheral cartilaginous tumor behavior cannot be ensured. Consequently, an initial step in the process of defining histological parameters for guiding the diagnosis of peripheral cartilaginous tumors in the setting of multiple osteochondromas is to assess diagnostic reliability, as measured by intraclass correlation coefficient. ${ }^{13} \mathrm{~A}$ second step is to identify common histological criteria among the concordant cases, aiming to have histological parameters that characterize each tumor type. This study evaluated diagnostic concordance among a panel of experienced bone-tumor pathologists. Histological parameters noted to be of diagnostic value in the literature were then systematically evaluated among the concordant cases to give the best guidance on diagnosing peripheral cartilaginous tumors in the setting of multiple osteochondromas.

\section{Materials and methods}

\section{Cases Studied}

Patients with multiple osteochondromas admitted to Leiden University Medical Center between 1985 and 2009 with either osteochondroma or secondary peripheral chondrosarcoma were retrieved from our database. Cases with neither radiological documentation nor clinical information were excluded as well as cases with local recurrences and not enough material for histological examination. All the original diagnoses were made at the Leiden University Medical Center. In addition, 20 cases were further confirmed in multidisciplinary discussions organized by the Netherlands Committee on Bone Tumors, a national multidisciplinary committee for consultation on diagnosis and treatment of musculoskeletal tumors and tumor-like lesions.

Medical records were studied to identify patient age, gender and tumor location. Imaging studies, including plain radiographs, magnetic resonance images and/or computed tomography scans, were available in 29 cases. The cartilage cap thickness was measured by a bone-tumor radiologist (HMK) in accordance with Bernard et al. ${ }^{11}$ Briefly, the cap thickness was assessed by measuring the thickest portions of the cartilage cap perpendicular to the boundary between the medullary space of the osteochondroma stalk. For each case, at the minimum one complete section through the whole lesion including the maximal diameter of the cartilaginous cap was submitted for histology. Representative histological sections were subsequently selected for each case and were randomly labeled from 1 to 38. All the unique identifiers were removed to protect patient anonymity. The study was performed according to the ethical guidelines in the Code for Proper Secondary Use of Human Tissue in the Netherlands (Dutch Federation of Medical Scientific Societies).

\section{Interobserver Variability}

Case series reviewers were 12 pathologists (CE A; SR; AER; BRD; BLA; CYI; EH; EFM; MAIG; NAA; PCWH and JVMGB) selected for their expertise in bone-tumor pathology. The case series consisted of (1) an excel file with clinical information, in which the diagnosis, tumor grade and any possible remarks were asked to be filled in, (2) digitized imaging studies and (3) glass slide from each case. Many pathologists classified malignant secondary peripheral cartilaginous lesions into grade-I, -II or -III. Few 
reviewers used grades of low-grade malignant or high-grade malignant tumors. To have a common classification, grade-I tumors were considered lowgrade malignant and grade-II and -III were considered as high-grade.

\section{Histopathology}

The most common criteria noted to be of diagnostic value in the literature to distinguish osteochondroma, low-grade secondary peripheral chondrosarcoma, high-grade secondary peripheral chondrosarcoma and de-differentiated chondrosarcoma were evaluated among the concordant cases by three pathologists (CEA, JVMGB and PCWH). Concordant cases were defined as those that had an agreement of greater than $75 \%$ among the 12 pathologists. The list of histological parameters included: cellularity (highor low cellular), the presence/absence of binucleated cells, nuclear pleomorphism, calcification or irregular mineralization, nodularity, permeation of trabecular bone, cystic/mucoid changes, necrosis and mitotic figures., ${ }^{3,12}$

Histological parameters were defined as follows:

- Cellularity: High cellularity was defined as a cellrich lesion with closely packed cells with scant extracellular matrix in between. Low cellularity was defined as a matrix-rich lesion in which the cells were more distant from each other.

- Binucleated cells were defined as having two nuclei of a normal size sharing the same cytoplasm.

- Nuclear pleomorphism was defined as variation in nuclear size (three times normal size) and shape.

- Irregular calcification was interpreted as coarse and irregular areas of calcification (Figure 1d).

- Nodularity was defined by the presence of nodule(s) connected with the main lesion and separated from each other by cellular fibrous septa (Figure 1a).

- Permeation of trabecular bone was defined by tumor filling up the trabecular marrow space entrapping pre-existing lamellar bone trabeculae. ${ }^{14}$

- Cystic/mucoid changes were defined as areas or cystic spaces containing mucoid material (Figure 1b).

- Necrosis was identified by the presence of necrotic chondrocytes appearing as nuclei loosing hematoxylin staining (Figure 1c).

- Presence of mitotic figures was recorded whenever any mitotic figures were seen in any field at any rate.

\section{Statistical Analysis}

Intraclass correlation coefficients were calculated in a two-way random effects model to determine interobserver reliability among the 12 pathologists using the SPSS 16.0. software package (IBM,
Somers, NY, USA). Intraclass correlations are equivalent to weighted $\kappa$ coefficients with quadratic weights. ${ }^{13}$ Intraclass correlation coefficients were calculated for the agreement on diagnosis of osteochondroma, low-grade secondary peripheral chondrosarcoma, high-grade secondary peripheral chondrosarcoma and de-differentiated chondrosarcoma. Interpretation of intraclass correlation coefficients was performed according to Landis and Koch. ${ }^{15}$ A $\kappa$ value below 0.20 is considered poor, $0.21-0.40$ fair, $0.41-0.60$ moderate, $0.61-0.80$ substantial and $0.81-1.00$ very good. ${ }^{15}$ Oneway ANOVA was used to evaluate statistical differences of the cartilage cap thickness among the cases. Additionally, adjusted $\chi^{2}$ - test was used to determine whether there are significant differences between the histological criteria in each tumor type.

\section{Results}

Thirty-eight tumors from 29 patients were included in the study. Clinical information and thickness of the cartilage cap of the 38 cases are summarized in Figure 2. None of the studied patients showed adverse outcome (metastases or death of disease) up to the study period (range 24-312 months, mean 148.8). Two patients had local recurrence.

\section{Interobserver Variability in Diagnosis}

The intraclass correlation coefficient for interobserver reliability was 0.78 (95\% confidence interval: 0.694-0.859), which indicated substantial agreement. High diagnostic concordance (agreement $\geq 75 \%$ by all the reviewers) was observed in 31 cases (Figure 2). Among these concordant cases, 25 cases were diagnosed as osteochondroma, three cases as lowgrade secondary peripheral chondrosarcoma, two cases as high-grade secondary peripheral chondrosarcoma and one case as de-differentiated chondrosarcoma. Diagnostic concordance was not reached in seven cases (agreement $<75 \%$ by all the reviewers) (Figure 2). All the non-concordant cases were diagnosed by the Netherlands Committee on Bone Tumors as being low-grade secondary peripheral chondrosarcoma. Additionally, cases number 6 and 10 were described by the Netherlands Committee on Bone Tumors as progressing to highgrade lesions. Case 6 had a cartilage cap thickness of $6 \mathrm{~cm}$ and neither mitotic figures nor nuclear pleomorphism were found. Case 10 had a cartilage cap thickness of $10 \mathrm{~cm}$ and only nuclear pleomorphism was identified. These two cases had a local recurrence after surgery. One lesion (case 9) had a large spindle cell component, which was interpreted as de-differentiated chondrosarcoma by 10/12 pathologists. 

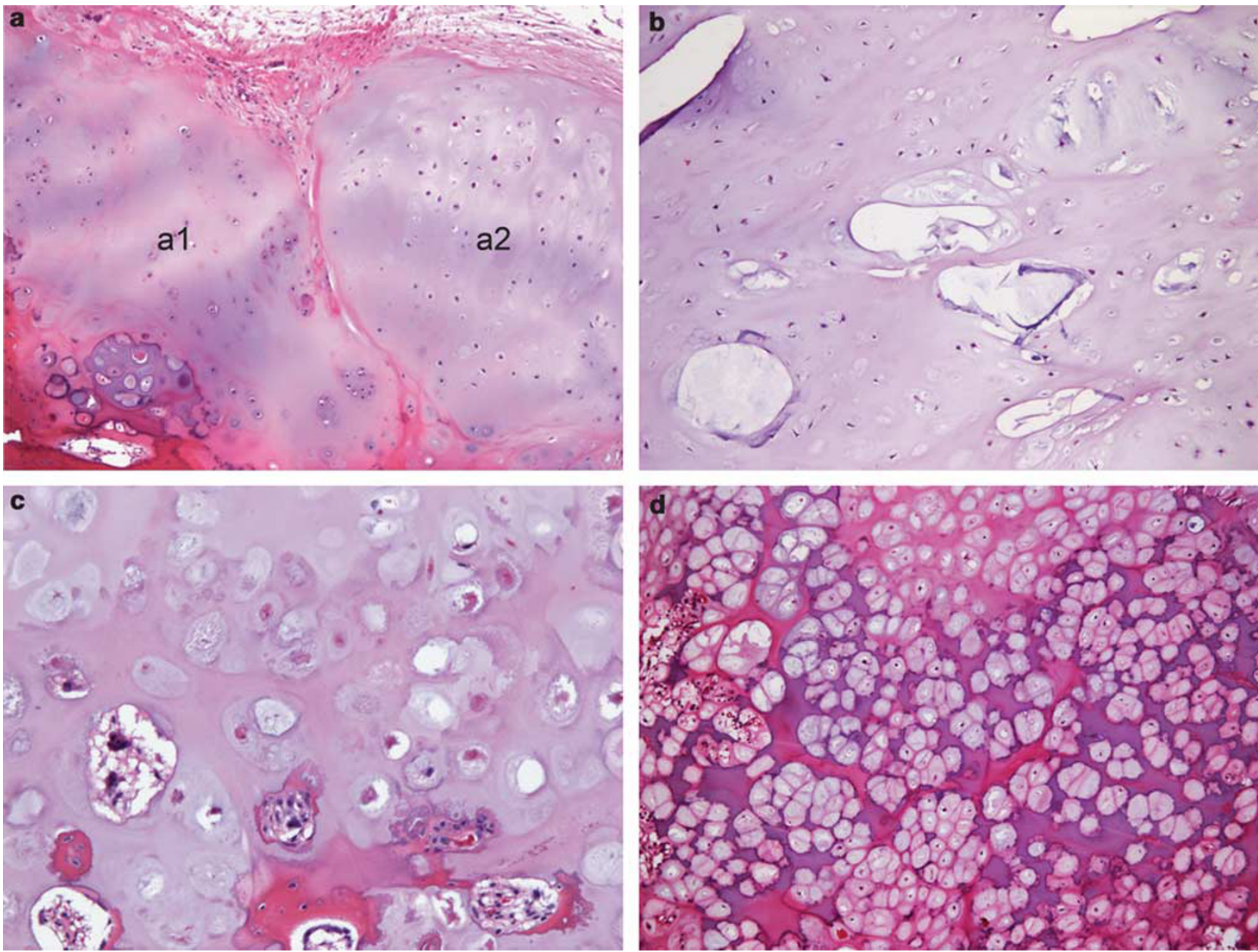

Figure 1 (a-c) Histological features often observed in lesions interpreted as osteochondromas. (a) Nodularity: a nodule is connected with the main lesion (a1) and separated from the main lesion by fibrous septa (a2). (b) Cystic changes (areas of cystic spaces containing mucoid material) are irregularly distributed in the osteochondroma cartilage cap. (c) Necrosis: necrotic chondrocytes appeared as swollen nuclei that lost haematoxylin staining. (d) Irregular calcification: coarse and irregular calcification is often seen.

\section{Diagnostic Value of Histological Parameters}

All concordant cases were systematically evaluated by three bone-tumor pathologists (CEA, JVMGB and PCWH) and data are shown in Table 1. Interestingly, among osteochondromas, binucleated cells, irregular calcification and necrosis were observed in $72 \%$ of the cases (Figures 1a-d). Additionally, nodularity and cystic/mucoid changes were identified in 56\% and $76 \%$ of the osteochondroma cases, respectively (Figures 1a and b). All these histological parameters were also seen in low- and high-grade secondary peripheral chondrosarcomas (Figures 3a-f). Characteristically, mitotic figures at any rate and nuclear pleomorphism were only observed in high-grade secondary peripheral chondrosarcoma. Singly or collectively, no significant differences were observed between the histological criteria in each tumor type (Table 1). Only the presence of mitotic figures and nuclear pleomorphism in high-grade secondary peripheral chondrosarcoma were signifi- cantly different compared with osteochondroma and low-grade secondary peripheral chondrosarcoma $(P<0.001)$. Permeation of trabecular bone was not observed in any case.

\section{Diagnostic Value of Cartilage Cap Thickness}

The average of cartilage cap thickness was $0.82 \mathrm{~cm}$ (ranged from 0.1 to $2 \mathrm{~cm}$ ) in osteochondromas, $3.83 \mathrm{~cm}$ (ranged from 2.5 to $5 \mathrm{~cm}$ ) in low-grade secondary peripheral chondrosarcomas and $5.50 \mathrm{~cm}$ (ranged from 2 to $9 \mathrm{~cm}$ ) in high-grade secondary peripheral chondrosarcomas. With regard to cartilage cap thickness among the concordant cases, a statistically significant difference was found in osteochondroma compared with low- and highgrade secondary peripheral chondrosarcoma $(P=0.001)$. In addition, among the concordant cases, no significant difference was seen between low- and high-grade secondary peripheral chondro- 


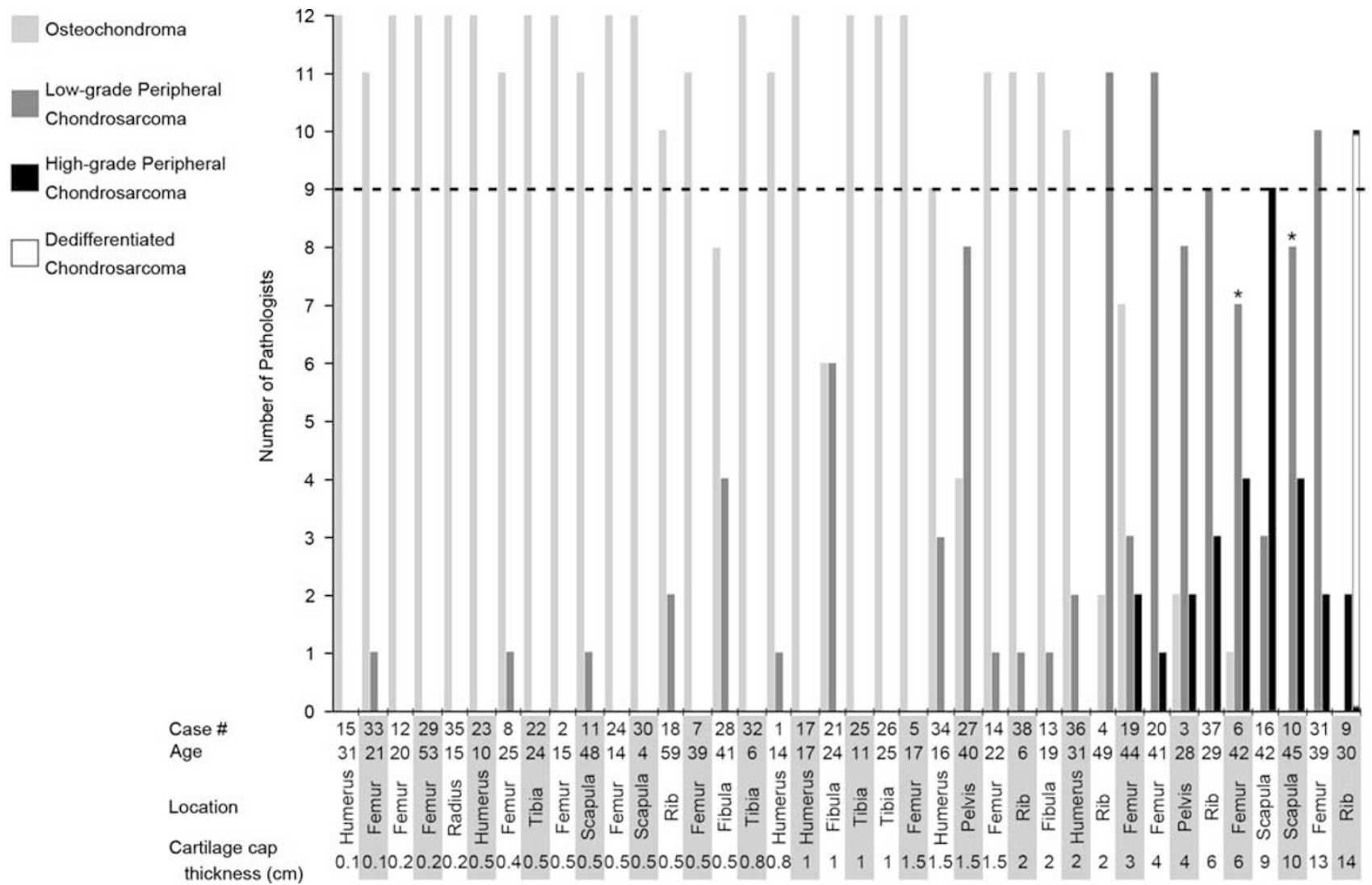

Figure 2 Chart displaying the raw data from pathologists and clinical details of the 38 cases to study interobserver reliability. Concordant cases show agreement among the 12 pathologists greater than $75 \%$ (dashed line). Asterisk indicates cases with local recurrence.

sarcoma $(P=0.272)$. Among the non-concordant cases (Figure 2), the average of cartilage cap thickness varied from 1 to $10 \mathrm{~cm}$.

\section{Discussion}

Analyses of a series of peripheral cartilaginous tumors in the setting of multiple osteochondromas by 12 experienced bone-tumor pathologists showed that there was a substantial agreement on diagnosis among all the reviewers. We here confirm that mitotic figures and nuclear pleomorphism are hallmarks of high-grade secondary peripheral chondrosarcoma, and that a malignant spindle cell component indicates de-differentiated peripheral chondrosarcoma.

We previously assessed interobserver variability in central cartilaginous lesions. ${ }^{16,17}$ Similar intraclass correlation coefficient was found for diagnosing central and peripheral lesions (in both 0.78). ${ }^{17}$ For central lesions, we were able to identify five parameters (high cellularity, presence of host bone entrapment, open chromatin, mucoid matrix quality and age above 45 years) that could optimally differentiate between enchondroma and low-grade central chondrosarcoma, of which mucoid/myxoid matrix degenerative changes and host bone entrapment were the most important. ${ }^{17}$ The present study, however, demonstrates that these criteria cannot be applied for cartilaginous tumors in the setting of multiple osteochondromas. In addition, we show that histology alone cannot distinguish osteochondroma from low-grade secondary peripheral chondrosarcoma as nodularity, the presence of binucleated cells, irregular calcification, cystic/ mucoid changes and necrosis were not helpful to indicate malignant transformation of an osteochondroma.

Instead, our results emphasize that evaluation of the size of the cartilage cap at radiology and gross pathology is crucial. Among the concordant cases, osteochondromas had a significantly thinner cartilage cap compared with secondary peripheral chondrosarcomas. This reinforces the general consensus of distinguishing osteochondroma from lowgrade secondary peripheral chondrosarcoma by cartilage cap thickness. ${ }^{11}$ A standardized measuring technique with computed tomography and magnetic resonance imaging has been described to assess the cartilage cap thickness. ${ }^{11}$

Histologically, nodularity was observed in about half of osteochondromas. Nodules in osteochondromas have previously been considered a feature of 
malignant transformation. ${ }^{12,18,19}$ Interestingly, cases 21, 27 and 28 showed disagreement on distinguishing benign from low-grade malignant. Although the thickness of the cartilage cap in these three cases is not increased (1, 1.5 and $0.5 \mathrm{~cm}$, respectively), they display histological and radiological features of nodularity. The presence of nodularity in these tumors possibly led some pathologists to interpret this as suspicious for low-grade sarcoma. On the other hand, the thin cartilage cap of case 28 has probably influenced the decision of most pathologists to sign it out as a benign lesion. Although treatment planning was beyond the scope of this study, the presence of nodularity on imaging may have been interpreted as a sign suspicious of malignant transformation leading to subsequent resection. In the magnetic resonance imaging scan, nodules are often associated with vascularized septa

Table 1 Histological parameters scored among the concordant cases

\begin{tabular}{|c|c|c|c|c|c|c|c|}
\hline & \multicolumn{2}{|c|}{$\begin{array}{l}\text { Osteochondromas } \\
\qquad(\mathrm{n}=25)\end{array}$} & \multicolumn{2}{|c|}{$\begin{array}{l}\text { Low-grade secondary } \\
\text { peripheral chondrosarcomas } \\
\qquad(\mathrm{n}=3)\end{array}$} & \multicolumn{2}{|c|}{$\begin{array}{l}\text { High-grade secondary } \\
\text { peripheral chondrosarcomas } \\
(\mathrm{n}=2)\end{array}$} & \multirow[t]{2}{*}{$\mathrm{P}$} \\
\hline & No. & $\%$ & No. & $\%$ & No. & $\%$ & \\
\hline Cellularity & & & & & & & 0.260 \\
\hline Low & 14 & 56 & 1 & 33 & 0 & & \\
\hline High & 11 & 44 & 2 & 67 & 2 & 100 & \\
\hline Binucleated cells & & & & & & & 0.401 \\
\hline Present & 18 & 72 & 3 & 100 & 2 & 100 & \\
\hline Absent & 7 & 28 & 0 & & 0 & & \\
\hline Nuclear pleomorphism & & & & & & & $<0.001^{\mathrm{a}}$ \\
\hline Present & 0 & & 0 & & 2 & 100 & \\
\hline Absent & 25 & 100 & 3 & 100 & 0 & & \\
\hline Calcification/irregular mineralization & & & & & & & 0.401 \\
\hline Present & 18 & 72 & 3 & 100 & NA & & \\
\hline Absent & 7 & 28 & 0 & & NA & & \\
\hline Nodularity & & & & & & & 0.176 \\
\hline Present & 14 & 56 & 3 & 100 & 2 & 100 & \\
\hline Absent & 11 & 44 & 0 & & 0 & & \\
\hline Permeation of trabecular bone & & & & & & & NA \\
\hline Present & 0 & & 0 & & 0 & & \\
\hline Absent & 25 & 100 & 3 & 100 & 2 & 100 & \\
\hline Cystic/mucoid changes & & & & & & & 0.472 \\
\hline Present & 19 & 76 & 3 & 100 & 2 & 100 & \\
\hline Absent & 6 & 24 & 0 & & 0 & & \\
\hline Necrosis & & & & & & & 0.401 \\
\hline Present & 18 & 72 & 3 & 100 & 2 & 100 & \\
\hline Absent & 7 & 28 & 0 & & 0 & & \\
\hline Mitosis & & & & & & & $<0.001^{\mathrm{a}}$ \\
\hline Present & 0 & & 0 & & 2 & 100 & \\
\hline Absent & 25 & 100 & 3 & 100 & 0 & & \\
\hline
\end{tabular}

NA, not evaluable.

${ }^{\mathrm{a}}$ Statistically significant (adjusted $\chi^{2}$ test).

Figure 3 (a-f) Lesion interpreted as low-grade secondary peripheral chondrosarcoma by the majority of the study pathologists (Case 31). (a) Antero-posterior conventional radiograph of the proximal femur. Ossifying and calcifying mass in close relation to the surface of the femur. Arrow indicates the stalk of the original osteochondroma. (b) Coronal T1-weighted magnetic resonance imaging after intravenous administration of contrast. Large soft-tissue mass anterior and lateral to the femur with a serpentine pattern of enhancement characteristic of a chondromatous tumor, in this case the soft-tissue extension of a secondary chondrosarcoma arising from an osteochondroma. (c) Axial T1-weighted, magnetic resonance imaging after intravenous administration of contrast shows a secondary chondrosarcoma arising from an osteochondroma. The stalk of the original osteochondroma arises from the latero-posterior surface the proximal femur. Large soft-tissue mass, lateral and anterior of the femur with a serpentine pattern of enhancement characteristic of a chondromatous tumor. (d) Large lobules of cartilage contained closely arranged cells in lacunae define the lesion. (e, f) Cystic changes are often observed, and no nuclear pleomorphism or mitotic figures are seen. 

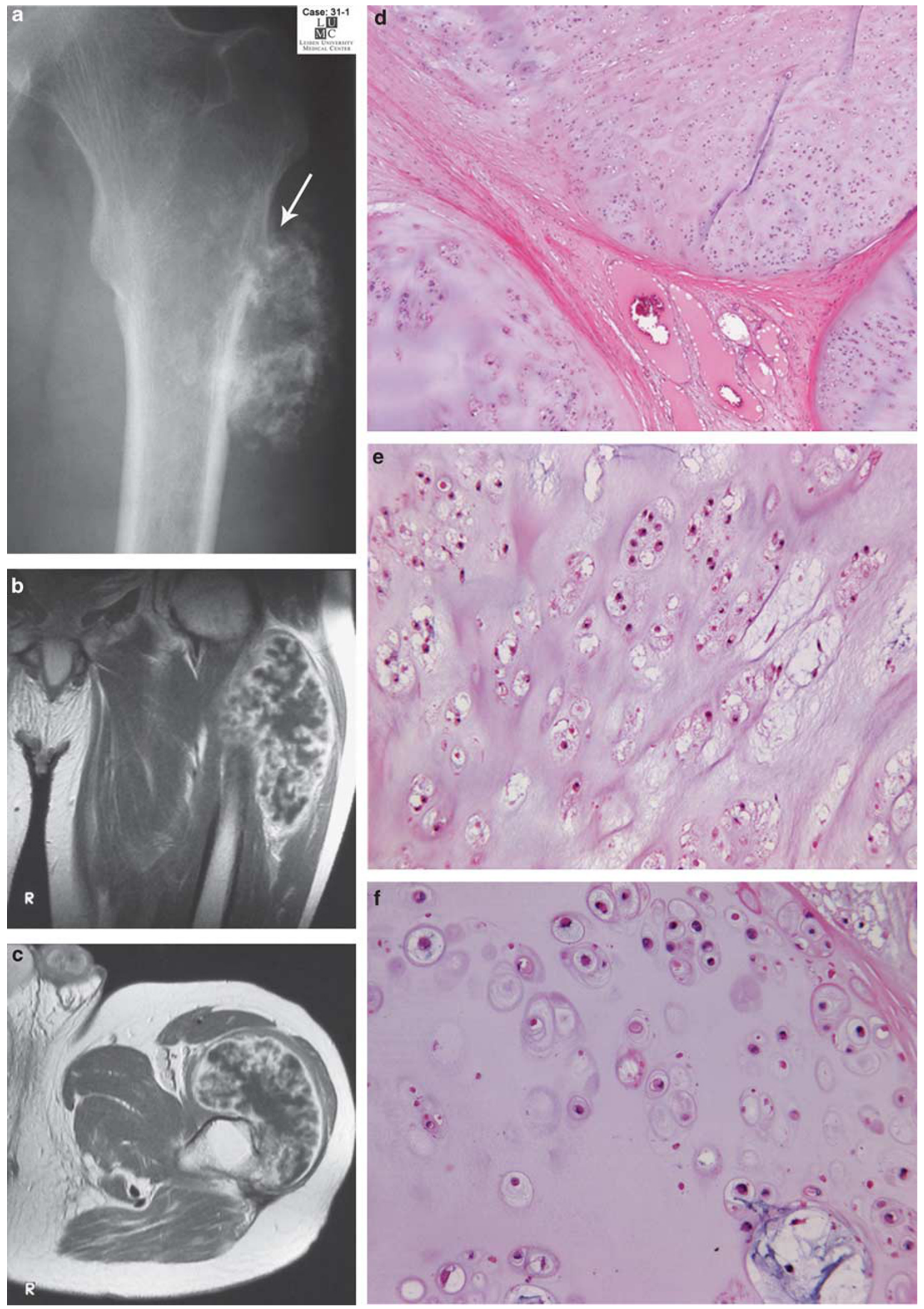
with high-intensity signal and are found in lowgrade chondrosarcomas and in active cartilaginous lesions. ${ }^{17}$

Considering that Leiden University Medical Center is a tertiary hospital for treatment of bone tumors in the Netherlands, we cannot exclude that our series is slightly biased toward lesions that are more prone to malignant transformation. The major limitation of our study is the lack of 'gold-standard' to distinguish osteochondroma from low-grade secondary peripheral chondrosarcoma, which has hampered the case selection. Low-grade secondary peripheral chondrosarcomas rarely metastasize and high-grade secondary peripheral chondrosarcomas are extremely rare. A selection of a set of cases with an equal ratio of benign, low- and high-grade tumors was therefore not feasible. Despite their rarity, we showed that high-grade secondary peripheral chondrosarcomas were distinguished from low-grade lesions by the presence of mitotic figures at any rate and nuclear pleomorphism, which is in line with previous literature. ${ }^{12,18}$

Histologically, osteochondroma and low-grade secondary peripheral chondrosarcoma in the setting of multiple osteochondromas were not distinguished by the presence of binucleated cells, irregular calcification, cystic/mucoid changes and focal necrosis. It has been described that osteochondromas occasionally have binucleated cells. ${ }^{3}$ Radiographically, areas of calcification in osteochondromas become disorganized and irregular, as the patient grows older. ${ }^{10}$ Cystic/mucoid changes are related to degenerative processes, such as mucinous degeneration. Similar degenerative processes have been described in osteoarthritis. ${ }^{18}$ Necrosis most likely is secondary to ischemia, as the cartilage cap is not vascularized and often traumatized. ${ }^{20}$

Sporadic peripheral cartilaginous tumors are histologically indistinguishable from peripheral cartilaginous tumors occurring in the setting of multiple osteochondromas. ${ }^{21}$ It is therefore most likely that we can extrapolate our findings indicating that histology alone is unable to confidently differentiate sporadic osteochondroma from lowgrade sporadic secondary peripheral chondrosarcomas as well.

Thus, our data indicate that a multi-disciplinary team, including pathologists, radiologists and orthopedic surgeons, is critical to establish a final diagnosis in sporadic and multiple peripheral cartilaginous tumors. Suspicious signs for malignancy in osteochondromas include tumor growth after pubertal growth spurt and pain. Osteochondromas located in the pelvis, hips and shoulders more often undergo malignant transformation. ${ }^{18}$

On plain radiographs, malignant progression of osteochondroma is suggested when osteolysis or change of chondroid calcification is observed. ${ }^{10}$ In addition, computed tomography scan might reveal new osteolytic areas and a change in the nature of calcifications in the periphery of osteochondromas. ${ }^{20}$
Moreover, soft-tissue swelling containing calcification is very suggestive of the development of a secondary peripheral chondrosarcoma on plain radiographs and computed tomography.

The cartilage cap of peripheral cartilaginous tumors is best evaluated by magnetic resonance imaging with T2-weighted images using fat-selective presaturation, which allows standardized measurements of the cap. A cartilage cap thickness greater than $2 \mathrm{~cm}$ in a skeletally mature patient is suspicious for neoplastic transformation of osteochondroma. ${ }^{11}$ Additionally, magnetic resonance imaging is used to delineate the extent of soft-tissue extension and its relation to the surrounding structures, specifically the neurovascular bundle. ${ }^{10}$ Contrastenhanced magnetic resonance imaging sequences are used to differentiate a thickened cartilage cap from an overlying bursa.

Whole-body bone scintigraphy in adult patients with multiple osteochondromas shows that lesions with increased uptake of the tracer may indicate malignant transformation. ${ }^{22}$

In summary, a review of 38 cases of peripheral cartilaginous tumors by 12 experienced bone-tumor pathologists showed general agreement in the diagnosis of these tumors in the setting of multiple osteochondromas. Histological parameters generally associated with malignant transformation could not reliably distinguish osteochondroma from low-grade secondary peripheral chondrosarcoma. Instead, a multidisciplinary approach integrating clinical and radiographical features and the size of the cartilaginous cap in combination with a histological assessment are crucial to the diagnosis of peripheral cartilaginous tumors.

\section{Acknowledgements}

We thank the Netherlands Committee on Bone Tumors, Dr PDS Dijkstra, Dr E Dootjes, and the expert technical work of Inge Briaire-de Bruijn. This study was supported by EuroBoNeT (a European Commission-granted European Network of excellence for studying the pathology and genetics of bone tumors, Grant Number: LSHC-CT-2006018814). JVMGB is financially supported by the Netherlands Organization for Scientific Research (Grant Number: 917-76-315).

\section{Disclosure/conflict of interest}

The authors declare no conflict of interest.

\section{References}

1 Schmale GA, Conrad EU, Raskind WH. The natural history of hereditary multiple exostoses. J Bone Joint Surg (Am) 1994;76:986-992. 
2 Bovee JVMG, Hogendoorn PCW, Wunder JS, et al. Cartilage tumours and bone development: molecular pathology and possible therapeutic targets. Nat Rev Cancer 2010;10:481-488.

3 Khurana J, Abdul-Karim F, Bovée JVMG. Osteochondroma. In: Fletcher CDM, Unni KK, Mertens F (eds). World Health Organization Classification of Tumours. Pathology and Genetics of Tumours of Soft Tissue and Bone, 3th edn. IARC Press: Lyon, 2002, pp 234-236.

4 Dorfman HD, Czerniak B, Kotz R, et al. WHO classification of tumours of bone: Introduction. In: Fletcher CDM, Unni KK, Mertens F (eds). World Health Organization Classification of Tumours. Pathology and Genetics of Tumours of Soft Tissue and Bone, 3th edn. IARC Press: Lyon, 2002, pp 226-232.

5 Campanacci M, Enneking WF. Peripheral chondrosarcoma. In: Campanacci M (ed). Bone and Soft Tissue Tumors: Clinical Features, Imaging, Pathology and Treatment, 2nd edn. Springer-Verlag: New York, 1999, pp 335-361.

6 Evans HL, Ayala AG, Romsdahl MM. Prognostic factors in chondrosarcoma of bone. A clinicopathologic analysis with emphasis on histologic grading. Cancer 1977;40:818-831.

7 Hogendoorn PC, Athanasou N, Bielack S, et al. Bone sarcomas: ESMO Clinical Practice Guidelines for diagnosis, treatment and follow-up. Ann Oncol 2010; 21:204-213.

8 Mulder JD, Schütte HE, Kroon HM, et al. The diagnosis of bone tumors. In: Mulder JD (ed). Radiologic atlas of bone tumors, 2nd edn. Elsevier: Amsterdam, 1993, pp 9-46.

9 Ahmed AR, Tan TS, Unni KK, et al. Secondary chondrosarcoma in osteochondroma: report of 107 patients. Clin Orthop Relat Res 2003;411:193-206.

10 Murphey MD, Choi JJ, Kransdorf MJ, et al. Imaging of osteochondroma: variants and complications with radiologic-pathologic correlation. RadioGraphics 2000; 20:1407-1434.

11 Bernard SA, Murphey MD, Flemming DJ, et al. Improved differentiation of benign osteochondromas from secondary chondrosarcomas with standardized measurement of cartilage cap at CT and MR imaging. Radiology 2010;255:857-865.

12 Bertoni F, Bacchini P, Hogendoorn PCW. Chondrosarcoma. In: Fletcher CDM, Unni KK, Mertens F (eds). World Health Organization Classification of Tumours.
Pathology and Genetics of Tumours of Soft Tissue and Bone, 3th edn. IARC Press: Lyon, 2002, pp 247-251.

13 Shrout PE, Fleiss JL. Intraclass correlations: uses in assessing rater reliability. Psychol Bull 1979;86:420-428.

14 Mirra JM, Gold R, Downs J, et al. A new histologic approach to the differentiation of enchondroma and chondrosarcoma of the bones. A clinicopathologic analysis of 51 cases. Clin Orthop 1985;201:214-237.

15 Landis JR, Koch GG. An application of hierarchical kappa-type statistics in the assessment of majority agreement among multiple observers. Biometrics 1977; 33:363-374

16 Skeletal Lesions Interobserver Correlation among Expert Diagnosticians (SLICED) Study Group. Reliability of histopathologic and radiologic grading of cartilaginous neoplasms in long bones. J Bone Joint Surg Am 2007;89:2113-2123.

17 Eefting D, Schrage YM, Geirnaerdt MJ, et al. Assessment of interobserver variability and histologic parameters to improve reliability in classification and grading of central cartilaginous tumors. Am J Surg Pathol 2009;33:50-57.

18 Unni KK. Chondrosarcoma (primary, secondary, dedifferentiated, and clear cell). In: Unni KK (ed). Dahlin's Bone Tumors: General Aspects and Data on 11,087 Cases, 5th edn. Lippincott-Raven Publishers: Philadelphia, 1996, pp 71-108.

19 Bloem JL, Van der Woude HJ, Hogendoorn PCW. MRI of the musculoskeletal system: a teaching file. In: Deutsch AL, Mink JH (eds). Bone Tumors, 2nd edn. LippincottRaven Publishers: Philadelphia, 1996, pp 655-719.

20 Carlson CS, Meuten DJ, Richardson DC. Ischemic necrosis of cartilage in spontaneous and experimental lesions of osteochondrosis. J Orthop Res 1991;9:317-329.

21 Ledingham JM, Preston BJ, Doherty M. Cystic change in the head of the fibula in osteoarthritis. J Bone Joint Surg Br 1993;75:429-432.

22 Romeo S, Hogendoorn PC, Dei Tos AP. Benign cartilaginous tumors of bone: from morphology to somatic and germ-line genetics. Adv Anat Pathol 2009; 16:307-315

23 Kenney PJ, Gilula LA, Murphy WA. The use of computed tomography to distinguish osteochondroma and chondrosarcoma. Radiology 1981;139:129-137.

24 Hendel HW, Daugaard S, Kjaer A. Utility of planar bone scintigraphy to distinguish benign osteochondromas from malignant chondrosarcomas. Clin Nucl Med 2002;27:622-624. 